\title{
Constraints and Defaults on Zero Pronouns in Japanese Instruction Manuals
}

\author{
Tatsunori Mori, Mamoru Matsuo, Hiroshi Nakagawa \\ Division of Electrical and Computer Engineering \\ Yokohama National University \\ 79-5 Tokiwadai, Hodogaya-ku, Yokohama 240, JAPAN \\ \{mori,mamoru\}@forest.dnj.ynu.ac.jp, nakagawa@naklab.dnj.ynu.ac.jp
}

\begin{abstract}
In this paper, we propose a method for anaphora resolution of zero subjects in Japanese manual sentences based on both the nature of language expressions and the ontology of ordinary instruction manuals. In instruction manuals written in Japanese, zero subjects often introduce ambiguity into sentences. In order to resolve them, we consider the property of several types of expressions including some forms of verbal phrases and some conjunctives of clauses, and so on. As the result, we have a set of constraints and defaults for zero subject resolution. We examine the precision of the constraints and defaults with real manual sentences, and we have the result that they make a good estimate with precision of over $80 \%$.
\end{abstract}

\section{Introduction}

From simple electrical appliances to complex computer systems, almost all machines are accompanied by instruction manuals. Since recently there are many machines whose operating procedures are complicated, we have much more trouble in many cases including translating their manuals into other languages, maintaining consistency between the description in manuals and the actual behavior of the machines. To solve these problems, we have to have a computer assisted system for processing manual sentences. In processing instruction manuals written in Japanese, however, it is problematic that almost all subjects are omitted. They are called "zero subjects." For example, machine translation systems have to supply appropriate subjects to translate sentences. Therefore, we have focused on anaphora resolution of zero subjects in Japanese manual sentences. Mori et al.(Mori and Nakagawa, 1996) show that properties of Japanese conditionals can be used to resolve them. In this paper, we propose new constraints and defaults based on properties of linguistic expressions, which are useful to estimate omitted subjects in addition to the constraints and defaults proposed by Mori et al.

A large number of researchers have come to grip with the method of understanding some types of text including instruction manuals(A be et al., 1988; Nomura, 1992; Eugenio, 1992). One of the most important matters of concern in these types of system is how we can resolve ambiguities in semantic representations and fill underspecified parts of them. Generally speaking, almost all systems described above take the following scheme. Firstly, each sentence in a text is translated into a semantic representation. In this process, the system uses only non-defeasible syntactic and semantic constraints. This way of analysis is known as the Nondefeasibility Thesis(Kameyama, 1995). Secondly, all of undetermined parts of the semantic representation are filled or settled by some kind of inferences based on the domain knowledge.

This type of method, which uses a large amount of domain knowledge, seems to be dominant from the viewpoint of disambiguation. Moreover it scarcely depends on the language in use because the way of disambiguation is based on the inference with a certain knowledge base. On the other hand, in order to use this method, we have to prepare the amount of knowledge being large enough to cope with various types of described objects. Unfortunately, so far we have not had such a commonsense knowledge base.

One of the ways to get rid of this situation is to adopt some knowledge which is independent of any particular domain. As such a kind of knowledge, we pay attention to pragmatic constraints, which have not been used sufficiently in the former methods. We expect that owing to pragmatic constraints the ambiguity in manual sentences would be resolved to some extent not in the process of inference but in the process of the translation of manual sentences into semantic representations.

We do not commit ourselves to the domain specific knowledge, but use some ontological knowledge of ordinary manuals. For example, the correspondence of objects in the manual sentences to the objects in linguistic constraints, namely linguistic roles like 
the speaker, the hearer, and so on. Note that the ontology in this paper does not refer to all of the objects in the world described by manuals, like a certain part of machine. Aiming at independence from the domain knowledge of objects, we adopt one of general ontologies which is applicable to almost all manuals.

Now we have to define the term 'SUBJECT' we used in this paper. Since our final goal is the determination of "the main participant" which is omitted, both of the term 'subject' and the term 'agent' are not suitable for referring to the omitted objects. For example, in a sentence in passive voice, the subject corresponds to not the agent(namely the main participant), but the patient. Moreover, there are several types of sentences whose subjects are main participants even if they are not agents, like the description of states, attributes and so on. Therefore, we use the term 'SUBJECT' to denote the main participant of the sentence, namely ether the agent or the surface subject(in the case where the agent is not defined).

\section{Zero pronouns in manual sentences}

Let's consider the following Japanese sentence, which shows a certain instruction.

(1) $\phi_{a}$ kono-botan-o osu -to, $\phi_{a}$-NOM this-button-ACC push -TO, $\phi_{b}$ der -are -mas -u.

$\phi_{b}$-NOM go out -can -POL -NONPAST.

If $\phi_{a}$ push(es) this button, then $\phi_{b}$ can go out.

Native speakers of Japanese have the following intuitive interpretation for (1) without any special context.

(2) $\phi_{a}=\phi_{b}=$ the hearer (= the user)

Here, 'TO' is a Japanese conjunctive particle which represents a causal relation, and 'ARE' shows ability or permission. The symbol $\phi$ denotes a zero pronoun.

On the other hand, the following sentence, which does not have the suffix 'ARE', has a different interpretation.

(3) $\phi_{c}$ kono-botan-o osu -to,

$\phi_{c}$-NOM this-button-ACC push -TO,

$\phi_{d} \quad$ de $\quad$ mas $-\mathrm{u}$.

$\phi_{d}$-NOM come out -POL -NONPAST. ${ }^{1}$

If $\phi_{c}$ push(es) this button, then $\phi_{d}$ will come out.

The zero pronoun $\phi_{d}$ refers to not the hearer(the user) but the machine, even though $\phi_{c}$ refers to the

\footnotetext{
'The English translation of 'DERU' in (3) is different from the translation in (1). It is due to the difference of the viewpoint between Japanese and English. The difference has no effect on the selection of zero pronoun's referent.
}

user as well as (1). Note that when only the matrix clause of (3) is used as shown in (4), $\phi_{e}$ can be interpreted as either the hearer or the machine ${ }^{2}$.

(4) $\phi_{e}$ de - mas $-\mathrm{u}$. $\phi_{e}$-NOM go out -POL -NONPAST. $\phi_{e}$ will go out.

These examples show that the expressions TO and ARE impose some constraints on the referents of SUBJECTS of the sentences. As described so far, there are many cases that linguistic expressions give us key information to resolve some type of ambiguity like the anaphora of zero pronouns. In the rest of this paper, we will show several pragmatic constraints, which can account for the interpretations of zero subjects including the cases described above.

Dohsaka(Dohsaka, 1994) proposes a similar approach, in which several pragmatic constraints are used to determine referents of zero pronouns. For example, honorific expressions and the speaker's point of view are used in his approach. While his approach treats dialogue, our targets are manual sentences.

Nakaiwa et.al.(Nakaiwa and Shirai, 1996) also propose the method which is based on semantic and pragmatic constraints. Although they report that their method estimates over $90 \%$ of zero subjects correctly, there are several difficulties including the fact that the test corpus is identical with the corpus from which the pragmatic constraints are extracted, and the fact that there are so many rules(46 rules to estimate 175 sentences).

As for the identifying method available in general discourses, the centering theory(Brennan et al., 1987; Walker et al., 1990) and the property sharing theory(Kameyama, 1988) are proposed. The important feature of these theories is the fact that it is independent of the type of discourse. However, according to our experimental result, it seems that these kinds of theory do not estimate zero subjects in high precision for manual sentences ${ }^{3}$. The linguistic constraints specific to expressions are more accurate than theirs if the constraints are applicable.

\section{$3 \quad$ Hypothesis and general ontology of manuals}

Kaiho et al.(Kaiho et al., 1987) explain the basic function of instruction manuals as follows:

- A manual is an interface between humans and machines based on language information.

\footnotetext{
${ }^{2} \mathrm{It}$ seems to be more natural that $\phi_{e}$ is interpreted as the hearer.

${ }^{3}$ The result precision of centering theory is $60 \%$ to $70 \%$ in our experiment. One reason why the precision is not so good is that the structure of texts in (Japanese) manuals is slightly different from the ordinary discourses structure.
} 
- Since the essential function of manuals is to provide users with information to make the machine operate properly, the existence of users should be considered at all times.

- Manuals should appropriately provide information which is required by users.

On the other hand, the following tendency are pointed out in many linguistic literatures.

- The readers have the same point of view as the writer.

- Generally, the first candidate of the point of view is the nominative.

According to these considerations, we make the following hypothesis:

\section{Hypothesis 1 (Manuals easy to understand)}

- All descriptions are written from the viewpoint of users. Therefore, in general, subjects in manual sentences tend to be users.

- Things users know, tend to be omitted for readability unless they are needed. Therefore, the subject of the sentence whose agent is a user tends to be omitted.

- On the other hand, things which readers do not known, like reactions of operations, prompt from machines and so on, tend to be specified explicitly.

As the parts of ontology, we should consider, at least, two types of information: the properties of the objects in manuals and the discourse situation that is characterized by linguistic roles like a writer and a reader.

\section{Constraint 1 (Objects) \\ User has intention. \\ Manufacturer has intention. \\ Machine has no intention. \\ Constraint 2 (Discourse Situation) \\ Speaker(Writer) $=$ Manufacturer \\ Hearer(Reader) $=U$ ser}

From these constraints of the ontology, we can obtain the constraint of persons as follows.

\section{Constraint 3 (Persons)}

First Person $=$ Manufacturer
Second Person $=$ User
Third Person $=$ Machine

In the rest of this paper, we will propose several constraints and defaults based on the property of linguistic expression under the hypothesis and the constraints described above. Then, we will examine them with test examples from several manual sentences. Note that the constraints and defaults we propose here are derived not from some specific manuals but from our linguistic consideration for each of linguistic expressions. Therefore, we do not adopt strict validation method like 'cross validations', which is used in machine learning, to examine them. However, in order to confirm the validity of our constraints and defaults, we have checked them out with 24 manuals from various areas. Although we cannot explain all of our defaults and constraints here because of shortage of space, we will briefly show the table of our all defaults and constraints in Section 6.

\section{Constraints and defaults based on the type of verbs}

\subsection{Request form}

The speaker uses the sentences in the request form or the solicitation form to prompt hearers to do the action described by the sentence. Therefore,

Constraint 4 (SUBJECT of sentence in the request form)

$A$ SUBJECT of a sentence in either the request form or the solicitation form is the hearer.

The combination of this constraint and Constraint 3 (Persons) shows that the SUBJECT is the user in such a case. In example manuals, there are 123 sentences in the request form and all of them satisfy Constraint 4 .

\subsection{Modality expressions}

Manual sentences may have a kind of modality expressing permission, ability, obligation, and so on. Sentences which have the expressions of ability or permission mean not only that it is possible for the SUBJECT to do the action, but also that the SUBJECT has his/her own choice of whether to do the action or not. Therefore,

Constraint 5 (SUBJECT of sentence with ability expressions)

$A$ SUBJECT of a sentence with the expressions of ability or permission must have his/her intention to make a choice about the action described by the sentence.

This constraint and Constraint 1 (Objects) show that a SUBJECT of a sentence with the expressions of ability or permission is a user, because all of the actions of manufacturer have been finished when the user is reading the manual. In example manuals, there are 56 sentences with the ability expressions and all of them satisfy Constraint 5 .

\subsection{RU form}

In Japanese, simple operation procedures are often described as simple sentences with no subjects whose verbs are of one of the following types: the RU form, the request form or the solicitation form. The RU form is the basic form of verbs and it denotes the non-past tense. Since the RU form has a neutral 
meaning, it does not impose any restriction on the SUBJECT. However, with Hypothesis 1 we expect that the zero subject tends to be a user.

Default 1 (SUBJECT of sentence with a verb in the RU form)

$A$ SUBJECT of a sentence with a verb in the $\mathrm{RU}$ form is a user.

In example manuals, there are 214 sentences with a verb in the RU form and with no subject, and the SUBJECTS of 172 sentences are users. Therefore, the precision of the default is about $80.4 \%$.

\subsection{Intransitives}

In almost all cases of machines which come with instruction manuals, their actions are initiated by some activities of users. The activities are represented not by intransitives but by transitives. Therefore, we expect that a SUBJECT of a sentence with an intransitive tends to be a machine.

Default 2 (SUBJECT of sentence with an intransitive)

A SUBJECT of a sentence with an intransitive is a machine.

In example manuals, there are 238 sentences with intransitves, and the SUBJECTS of 211 sentences are machines. Therefore, the precision of the default is about $88.7 \%$.

\subsection{Passives}

The passivization is the transfer of the viewpoint of the speaker from the nominative to the objective by exchanging their positions. Namely, the passivization is used to bring the objective in the active voice to readers' attention, when SUBJECT is not so important for readers. Since readers, or users, do not have to know what SUBJECT is, it is hard for a SUBJECT of a sentence in passive voice to be a user.

Default 3 (SUBJECT of passives)

$A$ SUBJECT of a passive is a machine.

In example manuals, there are 48 passives and the SUBJECTS of 46 sentences are machines. Therefore, the precision of the default rule is about $95.8 \%$.

\subsection{Causatives}

Since a causative expresses an event that the SUBJECT of the causative makes someone(or something) do some action, the SUBJECT should have some intention and the initiative in controlling someone's action. Since a user has the initiative, we propose the following default.

Default 4 (SUBJECT of causatives)

$A$ SUBJECT of a causative is a user.

In example manuals, there are 38 passives and the SUBJECTS of 36 sentences are machines. Therefore, the precision of the default rule is about $94.7 \%$.

\subsection{Expressions with the suffix -DESU}

Expressions with the suffix -DESU are divided into two groups:

- noun + the suffix of copula

- Adjective verb

Each of them expresses that a SUBJECT has some property. Since it is unusual to describe user's property in manuals. Therefore,

Default 5 (SUBJECT of sentence with the suffix -DESU)

$A$ SUBJECT of a sentence with the suffix-DESU is a machine.

In example manuals, there are 25 sentences with the expression, and all SUBJECT 's of them are machines.

\section{Constraints and Defaults based on types of Connectives}

\subsection{Conditionals}

Japanese has four conditional particles, TO, REBA, TARA and NARA, which are attached to the end of subordinate clauses as described in (1). The subordinate clause and the matrix clause conjoined by one of these particles correspond to the antecedent and the consequence, respectively. The difference of constraints of these expressions are shown in the following sentences, which are the variants of the sentence (3).

(5) $\phi_{i}$ kono-botan-o ose -ba $\phi_{i}$-NOM this-button-ACC push -REBA, $\phi_{j}$ de -mas -u. $\phi_{j}$-NOM come out -POL -NONPAST.

If $\phi_{i}$ push(es) this button, then $\phi_{j}$ will come out.

(6) $\phi_{k} \quad$ kono-botan-o osi -tara, $\phi_{k}$-NOM this-button-ACC push -TARA, $\phi_{l}$ de -mas -u. $\phi_{l}$-NOM come out/go out-POL -NONPAST.

If $\phi_{k} \operatorname{push}(\mathrm{es})$ this button, then $\phi_{l}$ will come out/go out.

(7) $\phi_{m} \quad$ kono-botan-o osu -nara, $\phi_{m}$-NOM this-button-ACC push -NARA, $\phi_{n}$ de - mas -u. $\phi_{n}$-NOM come out/go out -POL -NONPAST.

If $\phi_{m} \operatorname{push}(\mathrm{es})$ this button, then $\phi_{n}$ will come out/go out.

As well as the sentence (3), for Japanese native speakers, the SUBJECT of the matrix clause of (5) should be a machine. On the other hand, in the case of the sentences (6) and (7), the SUBJECTS of the matrix clauses can be either users or machines. These phenomena probably due to the nature of each conditionals(Masuoka, 1993). Since a causal relation, which is shown by TO or REBA, expresses a general rule, the consequence cannot include speaker's 
attitude, like volition and request. Therefore, the SUBJECT of the matrix clause should be a machine. In contrast, in the case of assumptions, that is TARA and NARA, there are no such restrictions on the SUBJECT .

Based on these observation, Mori et al. (Mori and Nakagawa, 1995; Mori and Nakagawa, 1996) propose the defaults of SUBJECTS of sentences with these conditionals. Since it depends on the volitionality of the verb whether a sentence shows a speaker's attitude or not, the constraint and defaults are described in terms of volitionality of each verb. Note that the electronic dictionary IPAL provides the information of volitionality for each Japanese verb entry(IPA Technology center, 1987). According to the classification by IPAL, all of Japanese verbs are classified into two types, volitional verbs, which usually express intentional actions, and nonvolitional verbs, which express non-intentional actions. Although non-volitional verbs only express non-volitional actions(non-volitional use), some of volitional verbs have not only volitional use but also non-volitional use.

\section{Default 6 (SUBJECT of sentence with TO or REBA)}

The matrix clause does not express user's volitional action. Therefore, the SUBJECT of the matrix clause is a machine, if the verb of the matrix clause does not have the non-volitional use.

Default 7 (SUBJECT of sentence with TARA or NARA)

The matrix clause expresses only user's volitional action. Therefore, the SUBJECT of the matrix clause is a user.

The precision of the default rules of TO,REBA,TARA and NARA is $100 \%, 95.1 \%, 89.8 \%$ and $100 \%$, respectively.

\subsection{Adverbial conjunctive forms}

Japanese verbs have two major adverbial conjunctive forms: '-TE form' and 'adverbial form.' Roughly speaking, a clause with a verb in one of these forms is placed in front of another clause and they construct a coordinate relation. The following example shows the coordination of -TE form.

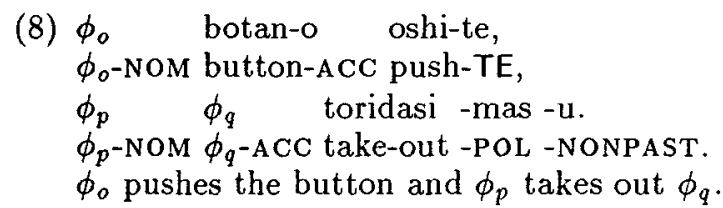

According to Teramura(Teramura, 1991), essentially these forms of verbs express the coordination and cooccurrence of two events. For example, the most plausible interpretation of (8) is that $\phi_{o}$ and $\phi_{p}$ are identical. Thus it is expected that two SUBJECTS of two clause in the coordination are identical or of the same type. Especially in manuals, the writer does not describe user's actions in the same treatment as machine's action, because the writer takes the viewpoint of users as supposed in Hypothesis 1. Therefore,

Default 8 (Two SUBJECTS of clauses in TE form conjunction or adverbial form conjunction)

Two SUBJECTS of two clauses are identical when the two clauses are connected by the TE form conjunction or the adverbial form conjunction.

In example manuals, there are 83 sentences with TE form conjunction and $\overline{7 j}$ sentences meets the default. Thus the precision of the default for sentences with TE form conjunction is about $90.4 \%$. Similarly, there are 99 sentences with adverbial form conjunction and 98 sentences complies the default. The precision of the default for adverbial form conjunction is about $99.0 \%$. Moreover, in the majority of the cases of TE form conjunctions, SUBJECT is a user ( 85 cases). Therefore we revise the default for adverbial form conjunction as follows.

Default 9 (Two SUBJECTS of clauses in TE form conjunction)

Each SUBJECTS of two clauses is a user when the clauses are connected by the TE form conjunction.

\section{Results}

The constraints and defaults we proposed here and their precision are summarized as Table 1.

Note that this table shows that each default rule is strong enough for anaphora resolution of zero SUBJECT if it is applicable. Therefore, we have to examine the total performance of our method, that is, have to verify what percentage of zero SUBJECTS in manuals are correctly determined with our defaults. In order for the verification, we examined the estimate by our defaults with 9 test example manuals, which contain 740 sentences. Table 2 shows the result of the verification. Here, the term 'Restricted' shows that the candidate of SUBJECT is correctly restricted by Default 8 .

As the table shows, our defaults successfully determine zero SUBJECTS in manuals with the precision of over $80 \%$. It is remarkable that the rate of wrong judgment is only $2.5 \%$. Since almost all zero SUBJECTS which cannot be resolved by our defaults are still undetermined, it is possible to improve the precision by adding new defaults/constraint, combining other methods of zero pronoun resolution, and so on.

\section{Conclusion}

In this paper, we proposed a scheme which closely depends not on domain knowledge of objects described in manuals but on pragmatic constraints which linguistic expressions innately have. This method uses only the linguistic constraints and the general ontology of the world described by manuals. 
Table 1: Constraints and Defaults

\begin{tabular}{|c|r|c|r|}
\hline Expression & \# of occurrences & Estimation of SUBJ & Precision \\
\hline \hline Request & 123 & User & $100 \%$ \\
\hline Ability & 56 & User & $100 \%$ \\
\hline TO & 385 & Machine(Matrix) & $100 \%$ \\
\hline REBA & 225 & Machine(Matrix) & $95.1 \%$ \\
\hline TARA & 59 & User(Matrix) & $89.8 \%$ \\
\hline NARA & 9 & User(Matrix) & $100 \%$ \\
\hline $\begin{array}{c}\text { Simple } \\
\text { Non-past }\end{array}$ & 214 & User & $80.4 \%$ \\
\hline Intransitive & 238 & Machine & $88.7 \%$ \\
\hline Passive & 48 & Machine & $95.8 \%$ \\
\hline Causative & 38 & User & $94.7 \%$ \\
\hline 'Want to' & 4 & User & $100 \%$ \\
\hline Copura & 25 & Machine & $100 \%$ \\
\hline $\begin{array}{c}\text { 'Automat- } \\
\text { ically' }\end{array}$ & 20 & Machine & $100 \%$ \\
\hline TE-Conn. & 99 & User & $85.9 \%$ \\
\hline Adverbial-Conn. & 83 & Identical SUBJs & $90.4 \%$ \\
\hline
\end{tabular}

Table 2: Examination of our method

\begin{tabular}{|c|c|c|c|c|}
\hline \multirow{2}{*}{$\begin{array}{l}\text { Judgment by human } \\
\text { for zero subjects }\end{array}$} & \multicolumn{4}{|c|}{ Judgment by our method } \\
\hline & $\begin{array}{c}\text { Correct } \\
\text { (Subject is fixed) }\end{array}$ & $\begin{array}{c}\text { Correct } \\
\text { (Subject is 'restricted') }\end{array}$ & Wrong & $\begin{array}{c}\text { Not } \\
\text { applicable }\end{array}$ \\
\hline User & 548 & 24 & 6 & 114 \\
\hline Machine & 221 & 2 & 18 & 21 \\
\hline Manufacturer & 0 & 0 & 0 & 2 \\
\hline & $\overline{80.6 \%}$ & $2.7 \%$ & $2.5 \%$ & $14.2 \%$ \\
\hline
\end{tabular}

*Each figure shows the number of sentences. 
We have shown that we can determine the referents of zero pronouns to some extent with our linguistic constraints and defaults. However, we do not have enough knowledge about the following points. They are important portions of our future work.

- Utilization of discourse structure specific to manuals.

- Analysis for the other types of manual sentences, like definitions.

\section{References}

Norihiro Abe, Tomohiro Ishikawa, and Tsuji Saburo. 1988. Generating an assembly procedure from assembly instruction. Journal of Japanese Society for Artificial Intelligence, 3(5):590-598, September. (in Japanese).

Susan E. Brennan, Marilyn Walker Friedman, and Carl J. Pollard. 1987. A centering approach to pronouns. In Proceedings of the 25th Annual Meeting of the American Association of Computational Linguistics (ACL 87), pages 155-162.

Kohji Dohsaka. 1994. Identifying the referents of Japanese zero pronouns based on pragmatic condition interpretation. Transactions of Information Processing Society of Japan, 35(5):768-778. (in Japanese).

Barbara Di Eugenio. 1992. Understanding natural language instructions: The case of purpose clause. In Proceedings of $30 t h$ Annual Meeting of $A C L$, pages 120-127.

IPA Technology center, 1987. The lexicon of the Japanese basic verbs for Computers. Information-technology Promotion Agency(IPA), Japan, March. (in Japanese).

Hiroyuki Kaiho, Takashi Kato, Keizo Hori, and Etsuko Harada. 1987. User/Yomite-no kokoro-o tsukamu manual-no kaki-kata (How to write instruction manuals). Kyoritsu Pub., Tokyo. (in Japanese).

Megumi Kameyama. 1988. Japanese zero pronominal binding: Where syntax and discourse meet. In W. Poser, editor, Japanese Syntax, pages 351-369. CSLI,Stanford.

Megumi Kameyama. 1995. The syntax and semantics of the Japanese language engine. In R. Mazuka and N. Nagai, editors, Japanese Syntactic Processing. Lawrence Erlbaum Associates.

Takashi Masuoka, editor. 1993. Nihongo-no JokenHyogen (Conditional Expressions in Japanese). Kurosio Publishers, Tokyo. (in Japanese).

Tatsunori Mori and Hiroshi Nakagawa. 1995. A pragmatic approach to zero pronoun resolution in Japanese manual sentences - the case of
Japanese conditionals - In Proceedings of Natural Language Processing Pacific Rim Symposium '95 (NLPRS'95), pages 296-301, December.

Tatsunori Mori and Hiroshi Nakagawa. 1996. Zero pronouns and conditionals in Japanese instruction manuals. In Proceedings of the 16th International Conference on Computational Linguistics (COLING 96), pages 782-787, August.

Hiromi Nakaiwa and Satoshi Shirai. 1996. Anaphora resolution of Japanese zero pronouns with deictic reference. In Proceedings of the 16th International Conference on Computational Linguistics (COLING 96), pages 812-817, August.

Hirosato Nomura. 1992. Linguistic analysis of law sentences. In Proceedings of the 6th International Symposium: Legal Knowledge and Legal Reasoning Systems, pages 10-18. Legal Expert System Association, October.

Hideo Teramura. 1991. Nihongo-no sintakusu-to imi III (Syntax and Semantics of Japanese III). Kuroshio shuppan Pub. (in Japanese).

Marilyn Walker, Masayo Iida, and Sharon Cote. 1990. Centering in Japanese discourse. In Proceedings of COLING 90. 